\title{
The Midwest Exercise Trial for the Prevention of Weight Regain: MET POWeR
}

\author{
Joseph E. Donnellya, ${ }^{\star}$, Richard A. Washburna ${ }^{a}$, Debra K. Sullivan ${ }^{b}$, Jeffery J. Honas ${ }^{a}$, \\ Matthew S. Mayo ${ }^{c}$, Jeannine Goetz ${ }^{b}$, Jaehoon Lee ${ }^{a}$, and Amanda N. Szabo ${ }^{a}$ \\ ${ }^{a}$ Cardiovascular Research Institute, Division of Internal Medicine, The University of Kansas \\ Medical Center, 3901 Rainbow Boulevard, Kansas City, KS, 66160 USA \\ bepartment of Dietetics and Nutrition, The University of Kansas Medical Center, 3901 Rainbow \\ Boulevard, Kansas City, KS, 66160 USA \\ 'Department of Biostatistics, The University of Kansas Medical Center, 3901 Rainbow Boulevard, \\ Kansas City, KS, 66160 USA
}

\begin{abstract}
Weight reduction in overweight and obese individual's results in physiological and behavioral changes that make the prevention of weight regain more difficult than either initial weight loss or the prevention of weight gain. Exercise is recommended for the prevention of weight regain by both governmental agencies and professional organizations. To date, the effectiveness of exercise recommendations for the prevention of weight regain has not been evaluated in a properly designed, adequately powered trial. Therefore, we will conduct a randomized trial to evaluate the effectiveness of 3 levels of exercise on the prevention of weight regain, in initially overweight and obese sedentary men and women. Participants will complete a 3 month weight loss intervention of decreased energy intake (EI) and increased exercise (100 minutes/week). Participants achieving clinically significant weight loss ( $\geq 5 \%$ of initial weight), will then be randomly assigned to 12 months of verified exercise at 3 levels $(150,225$ or 300 minutes/week). This study will evaluate: 1) the effectiveness of 3 levels of exercise on the prevention of weight regain over 12 months subsequent to clinically significant weight loss $(25 \%) ; 2)$ gender differences in weight regain in response to 3 levels of exercise; and 3) potential compensatory changes in daily physical activity (PA) and EI on weight regain in response to 3 levels of exercise. Results of this investigation will provide information to develop evidenced based recommendations for the level of exercise associated with the prevention of weight regain.
\end{abstract}

\section{Keywords}

aerobic exercise; weight management; weight regain; obesity; gender; energy expenditure

\footnotetext{
(C) 2013 Elsevier Inc. All rights reserved.

*Corresponding Author: Amanda N. Szabo, Robinson Center, Rm. 100, The University of Kansas-Lawrence 1301 Sunnyside Avenue Lawrence, KS 66045 Phone: 785-764-3770, Fax: 785-864-2009, aszabo2@ku.edu.

Co-author emails: Joseph E. Donnelly (jdonnelly@ku.edu) Richard A. Washburn (rwashburn@ku.edu) Debra K. Sullivan (dsulliva@kumc.edu) Jeffery J. Honas (jhonas@ku.edu) Jeannine Goetz (jgoetz@kumc.edu) Matthew S. Mayo (mmayo@ kumc.edu) Amanda Szabo (aszabo2@ku.edu)

Publisher's Disclaimer: This is a PDF file of an unedited manuscript that has been accepted for publication. As a service to our customers we are providing this early version of the manuscript. The manuscript will undergo copyediting, typesetting, and review of the resulting proof before it is published in its final citable form. Please note that during the production process errors may be discovered which could affect the content, and all legal disclaimers that apply to the journal pertain.
} 


\section{Introduction}

Numerous combinations of restricted energy intake (EI) and increased exercise energy expenditure (EEEx) have shown moderate short-term ( $<6$ mos.) success in producing clinically significant ( $25 \%$ ) [1-6] reductions in body weight [7-10]. However, the prevalence of overweight and obesity among US adults continues at approximately $69 \%$ [11], due in part to the inability of individuals who lose weight to maintain weight loss [8, 12-16]. The prevention of weight regain differs from both initial weight loss and from maintenance of normal weight. Weight loss increases the propensity for weight regain through decreases in sympathetic tone, leptin, insulin, and bioactive thyroid hormones, an increase in ghrelin and changes in substrate utilization which result in altered energy balance by impacting EI and energy expenditure [resting metabolic rate (RMR) and physical activity (PA)] [17-23].

Exercise has been recommended for the prevention of weight regain by both governmental agencies and professional organizations including the International Association for the Study of Obesity (IASO) [24], the Institute of Medicine (IOM) [25], and the American College of Sports Medicine (ACSM))[26]. The US Department of Health and Human Services (HHS) 2008 report entitled "Physical Activity Guidelines for Americans" recommends $30 \mathrm{~min} / \mathrm{d}$ of moderate PA to elicit health benefits [27]. The HHS guidelines, although not specific to weight management have been interpreted to be sufficient for that purpose. Support for these recommendations is tenuous and is derived from studies with important limitations including: 1) cross-sectional, non-randomized observational designs $[2,15,28-33]$, randomization prior to weight loss: secondary analyses of exercise groups formed at study completion [34-36], self-reported levels of exercise [28] [36, 37] [38]; and 5) failure to supervise exercise and verify level of EEEx [36-38].

Three organizations offering exercise recommendations for the prevention of weight regain (HHS, IOM, ACSM) have highlighted the need to conduct adequately powered studies of sufficient duration, with randomization to different levels of exercise following completion of weight loss to address the issue of the amount of exercise required to minimize weight regain. Therefore, we will conduct a randomized trial to evaluate the effectiveness of 3 levels of exercise on the prevention of weight regain in a sample of initially overweight and obese sedentary men and women. Participants will complete a 3 month weight loss intervention consisting of decreased EI and increased PA (100 min/week). Participants achieving clinically significant weight loss ( $25 \%$ of initial weight), will then be randomly assigned to 12 months of exercise at either 150,225 or $300 \mathrm{~min} / \mathrm{week}$. This study will evaluate: 1) the effectiveness of 3 levels of exercise on the prevention of weight regain over 12 months subsequent to clinically significant weight loss ( $25 \%)$; 2) gender differences in weight regain in response to 3 levels of exercise; and 3) potential compensatory changes in daily PA and EI on weight regain in response to 3 levels of exercise.

\section{Materials and methods}

\subsection{Participants}

We will recruit 287 overweight or obese adults from Lawrence and the Kansas City, Kansas metropolitan area who will be compensated for their participation. The sample will include at least $50 \%$ women and $20 \%$ minorities. Primary care physician (PCP) clearance will be required for participation. The following inclusion/exclusion criteria will be used: Inclusion. 1) Age 21 to 55 years. We have restricted our sample to this age range because we believe that behavioral interventions for weight loss may be different for individuals who are younger or older than this proposed age range. Additionally, individuals above age 55 are likely to have a greater numbers of medical problems and medication use that could significantly impact the exercise protocol and outcomes. 2) BMI of $\geq 25.0$ to $45.0 \mathrm{~kg} / \mathrm{m}^{2}$. 
We have restricted our sample to this BMI range because individuals with a BMI less than $25 \mathrm{~kg} / \mathrm{m}^{2}$ are not classified as overweight and individuals with a BMI $>45.0 \mathrm{~kg} / \mathrm{m}^{2}$ may require more aggressive weight loss/prevention of weight regain interventions than we have proposed (e.g., surgery, medication, etc.). 3) Clearance for participation from their PCP. This will be obtained via a letter to the PCP that explains the research program and is returned to the investigators. Exclusion. 1) Participation in a research project involving weight loss or exercise in the previous 6 months, as these proximal experiences may impact the results of this study. 2) Participation in a regular exercise program (i.e., > $500 \mathrm{kcal} / \mathrm{week}$. of planned activity as estimated by questionnaire [39]. 3) Not weight stable $( \pm 4.5 \mathrm{~kg})$ for 3 months prior to intake as determined from an online initial eligibility questionnaire 4) Unwilling to be randomized to 1 of 3 exercise groups subsequent to weight loss. 5) Pregnant during the previous 6 months, lactating, or planned pregnancy in the following 15 months. 6) Serious medical risk such as type 1 diabetes, cancer or recent cardiac event (i.e., heart attack, angioplasty,etc.). Medical risk will be determined by a health history questionnaire and physician consent form. 7) Eating disorders as determined by a score of 20 or greater on the Eating Attitudes Test [40]. These individuals require counseling which is outside the scope of this study. 8) Current treatment for psychological problems, or taking psychotropic medications. Based on our experience, individuals meeting these criteria typically are problematic due to time conflicts with other treatments and medications which induce weight change. Addressing psychological problems is outside the scope of this study. 9) Taking medications known to significantly affect weight (gain or loss). 10) Adherence to specialized diet regimes, i.e., multiple food allergies, vegetarian, macrobiotic, etc. 11) Do not have access to grocery shopping and meal preparation (i.e. those in military, college with cafeteria plan, etc.). Approval for this study has been obtained from the Human Subjects Committee at the University of Kansas-Lawrence and the University of Kansas Medical Center-Kansas City.

\subsection{Randomization}

Randomization subsequent to weight loss is the only appropriate study design to adequately address our aims. Thus, only participants who achieve clinically significant weight loss ( $\geq$ $5 \%$ ) from baseline (-3 to 0 months) will be eligible for randomization for prevention of weight regain. Data from our previous weight loss trials indicate that $97 \%-99 \%$ of participants will meet the 5\% criteria for weight loss at 3 months and we expect $\sim 70 \%$ will achieve $\geq 10 \%$ weight loss over 3 months [41-43], thus providing a range of weight loss typical of many weight loss programs available to the public. A range of weight loss will also allow for for exploratory analyses to evaluate the impact of the amount of weight loss on weight regain in response to the exercise intervention. Participants will be stratified by gender and level of weight loss (i.e., 5 - 9.9\%, $10-14.9 \%, \geq 15 \%$ ) and will be sequentially randomized in a 1:1:1 allocation to 1 of 3 exercise groups. All randomization procedures will be completed by the project statistician. Those participants who achieve at least 5\% weight loss will then be notified on an individual basis regarding of their randomization assignment. Participants will be instructed not to share their randomization assignment with other participants and staff members associated with the study.

\subsection{Intervention: Behavioral weight loss clinics}

Sixty minute in-person behaviorally based clinics will be conducted weekly during the 3 month weight loss period (-3 to 0 months) and during the first 3 months of the prevention of weight regain intervention. During the final 9 months of weight regain prevention, clinics will be held twice per month using telephone conference calls. All clinics will use behavioral strategies based on Social Cognitive Theory (SCT) to promote change in both diet and exercise [44]. The following components of SCT will be employed: goal setting, self-monitoring, self-efficacy, manipulation of the environment to promote behavioral 
change, and reflection on outcome expectations and outcome value. Clinic sessions will begin with a check-in question designed to identify barriers to diet and exercise and to allow the group to work together to identify solutions which promotes group cohesion and social support. Weekly homework assignments are designed to increase self-efficacy for both diet and exercise and to provide practice of behavioral skills. For example, participants will be asked to identify items at the grocery store that meet calorie, fat, or fiber content consistent with a healthy diet. This learning experience improves confidence in the ability to identify food items to promote weight loss and prevent weight regain.

Clinics will be delivered by health educators trained and supervised by a co-investigator who will assure quality control and standardization of materials by conducting weekly staff meetings and reviewing tape recordings of clinic sessions. All clinic participants will receive a comprehensive program notebook which includes general guidelines regarding participation in the program, calendars and timelines for class meetings and data reporting, lessons for each clinic meeting, and detailed instructions for both the weight loss and prevention of weight regain diets, including appropriate recipes, handouts, worksheets, and assignments specific to the topic for each session.

During the last 9 months of the weight regain prevention intervention group phone clinics (conference calls) will be used instead of the traditional face-to-face clinic to reduce the burden of time and expense to participants and to maximize compliance with the intervention. The phone clinic (60 minute group conference call) will provide identical content and utilize the same procedures as the face-to-face clinic, with small differences for logistics associated with the conference call. At each phone or face-to-face clinic meeting, participants will record their consumption of pre-packaged meals (PM's-section 2.4),fruits and vegetables, and minutes of physical activity (both on and off site) and receive feedback, if needed, to improve dietary and physical activity compliance. Data on 295 participants from our recently completed effectiveness trial comparing weight management delivered by group phone conference vs. face-to-face (FTF) clinics suggests that weight change during the weight loss phase (baseline to 6 months) was $-13.4 \pm 6.7 \%$ and $-12.3 \pm 7.0 \%$ for FTF clinic and phone, respectively. Weight change during weight regain prevention (6 to 15 months) was $6.4 \pm 7.0 \%$ and $6.4 \pm 5.2 \%$, for FTF clinic and phone, respectively [45]. Results from this study and an earlier study by our group [43] suggest that phone delivery compared to FTF clinic meetings provides equivalent weight loss as well as maintenance. Therefore we are confident in our ability to deliver effective weight gain prevention clinics by group phone conference call as proposed for this study. An outline of clinic topics is presented in Table 1.

\subsection{Intervention: Weight loss diet ( -3 to 0 months)}

The weight loss and weight regain prevention diets for this study will be based on $25^{+}$years of experience with weight management [41-43, 46, 47]. EI will be reduced to $\sim 1,200$ to $1,500 \mathrm{kcal} /$ day using a combination of commercially available prepackaged meals (PM's), fruits and vegetables, low calorie shakes, and non-caloric beverages. Participants will consume a daily minimum of 2 entrees ( 180 to $270 \mathrm{kcals}$ each), at least 5 servings of fruits and/or vegetables, and 3 shakes ( 100 kcal each). Non-caloric beverages such as diet soda, coffee, etc. will be allowed ad libitum. When combined with a variety of fruits and vegetables, PM's (entrees + shakes) provide a diet with all necessary nutrients specified by the Dietary Reference Intakes [25].

We have targeted a minimum of 5\% weight reduction from baseline to 3 months; an amount of weight loss which is known to produce clinically significant reductions in risk for chronic diseases such as diabetes, heart disease, hypertension, and others [1-4]. In previous investigations by our group using a similar diet, weight loss at 3 months averaged $12.5 \%$ 
(range (11.1-13.7\%) [41, 43, 47]. Participants reaching a BMI of $22 \mathrm{~kg} / \mathrm{m}^{2}$ during weight loss will be transitioned to the prevention of weight regain diet described below; however, in our experience, this is an infrequent occurrence. Individuals achieving $<5 \%$ weight loss at 3 months will not be eligible for randomization and will be referred to our ongoing weight management programs or to other community resources for weight management.

\subsection{Intervention: Prevention of weight regain diet ( 0 - 12 months)}

We recognize there are numerous approaches to the prescription of EI for the prevention of weight regain. We were influenced by the IOM dietary recommendations for weight regain prevention [25], over 25 years experience with research trials and clinical weight management, and the desire to evaluate a practical and generalizable approach. Therefore, following weight loss we will recommend a daily EI of estimated RMR * 1.2 to account for activities of daily living [48]. This EI recommendation theoretically results in a negative energy balance in all groups when EEEx is considered. However, we anticipate compensatory changes in components of energy balance such as increased EI and/or decreased daily PA (both of which are measured in this trial) as the literature indicates most individuals regain weight subsequent to weight loss $[8,12-16]$. Thus, we believe our approach will maximize the potential to prevent weight regain and provides a reasonable compromise between scientific rigor, practicality, and generalizability.

During the weight regain prevention intervention, participants will receive a meal plan with suggested servings of grains, proteins, fruits, vegetables, dairy, and fats, based on their energy requirements and the USDA/HHS Dietary Guidelines for Americans 2010 [34]. Participants will be encouraged (not required) to continue consuming a minimum of 14 PM's (entrees or shakes) and a minimum of 35 servings of fruits and vegetables per week. They will be provided with a list of low calorie PM's and shakes available at local supermarkets or may purchase the PM's and shakes provided during the weight loss phase from the study coordinator.

\subsection{Intervention: Exercise during weight loss (-3 to 0 mos.)}

During weight loss we will use a verified, progressive exercise protocol that we have used in numerous previous investigations [41-43, 49]. Exercise will progress from 10 minutes/day, 5 days/week, $65 \%$ age-predicted maximal heart rate $\left(\mathrm{HR}_{\max }=220\right.$ - age in years $)$ to the goal of 20 minutes/day, 5/days/week, $70 \% \mathrm{HR}_{\max }$ (100 minutes/week) at week 7 and remain at this level through study week 12 . The intensity and duration of both on-site supervised and non-supervised exercise (walking or running) sessions will be verified by a HR monitor.

Participants will be required to complete a minimum of 3 of the 5 sessions under supervision in one of our 3 exercise facilities ( 2 in Lawrence, 1 in Kansas City). One of the supervised exercise sessions may be completed prior to/following the clinic meeting since the facilities for exercise and clinics are located in the same building. Each facility contains state-of-theart exercise equipment, televisions and provides an environment similar to a modern commercial exercise center. Treadmill walking will be the primary mode of supervised onsite exercise; however, we will permit alternative modes of aerobic exercise (elliptical, bike, etc.) during 1 exercise session/week. Allowing alternative exercise modes provides relief from the routine for some participants and may help prevent overuse injuries.

\subsection{Intervention: Exercise during prevention of weight regain (0 to 12 months)}

Subsequent to 3 months of weight loss, participants will be randomized to exercise groups of 150, 225 or $300 \mathrm{~min} /$ week. Exercise will be progressed from $100 \mathrm{~min} /$ week at $70 \%$ HRmax at the end of weight loss (week 12) to the prescribed goals over weeks 13-17 of weight regain prevention and remain at goal for the duration of the trial (weeks 18-60) (Table 2). 
Consistent with the protocol for exercise during weight loss ( -3 to 0 months) participants will be asked to complete a total of 5 exercise sessions/week and will be required to complete a minimum of 3 sessions under supervision in our exercise facilities. As during weight loss, treadmill walking will be the primary mode of on-site exercise; however, we will permit alternative modes of aerobic exercise (elliptical, bike, etc.) during 1 exercise session/week. We realize that these are demanding exercise protocols; however, they represent national recommendations for the level of exercise associated with the prevention of weight regain. Our group, as well as others, have demonstrated the ability of previously sedentary, overweight and obese individuals to adhere to these levels of exercise (185-300 $\mathrm{min} / \mathrm{wk}$ ) in trials of 10 [50], 12 [38] and $16 \mathrm{mo}$. [51] duration.

\subsection{Assessment of exercise compliance: Heart rate monitoring}

The frequency, intensity and duration of both supervised and unsupervised exercise sessions will be documented using downloadable HR monitors (Polar RS 400, Polar Electro Inc., Woodbury, NY) which are capable of collecting and storing 99 hours of HR data collected over 1-minute epochs. A valid exercise session will be defined as an average HR \pm 4 beats/ min of target HR for the prescribed exercise duration. Participants not completing a valid exercise session will be counseled to meet both the intensity and duration prescriptions.

Each exercise session will be preceded by a brief ( 5 minutes) warm up. Participants will then be instructed to increase their exercise intensity to reach their prescribed target HR (70\% of maximal HR \pm 4 beats/minute) prior to starting the HR monitor and to remain at the target intensity for the duration of each exercise session. During all supervised exercise sessions trained research staff will monitor participants exercise HR to insure that it remains at target. Prior to each supervised exercise session (minimum of 3 sessions/week) research staff will download HR data from all unsupervised exercise sessions completed since the last visit to the exercise laboratory using the Polar HR Software. Research staff will review intensity and duration of the unsupervised exercise sessions and provide feedback to the participant.

\subsection{Assessment of exercise compliance: Aerobic fitness}

As a secondary measure of compliance with the exercise protocol we will assesses aerobic fitness using a sub-maximal treadmill test at $-3,0,6$ and 12 months. The treadmill will be initially set a 3 m.p.h., $0 \%$ grade. Speed will remain constant and grade will be increased $1 \%$ each minute until the participant reaches either $75 \%$ age predicted $\mathrm{HR}_{\max }$ (participants not on beta blockers) or a rating of perceived exertion (RPE) of 16 on the Borg Scale (REF) (participants taking beta blockers). Heart rate (3-lead electrocardiogram: Marquette Electronics, Milwaukee, WI, USA) and perceived exertion will be assessed during the last 15 seconds of each stage. Aerobic fitness will be defined as the metabolic equivalent (MET) level estimated from treadmill speed and grade achieved during the last stage completed after achieving either the HR or RPE criteria.

\subsection{Assessment of EEEx}

Variation in body weight results in inter-individual differences in EEEx when performing weight bearing exercise of the same duration [51]. Therefore, to document the actual level of energy expenditure associated with each of the 3 exercise prescriptions (150, 225 and 300 minutes/week) over the course of the weight regain prevention trial we will assess EEEx in all participants at months 2, 7 and 12 to document the level of EEEx associated with each of the 3 exercise prescriptions. EEEx will be assessed by indirect calorimetry (ParvoMedics TrueOne2400, ParvoMedics Inc., Sandy, UT) during treadmill exercise over a 15 minute interval (1-minute epochs) at $75 \%$ age-predicted $\mathrm{HR}_{\max }( \pm 4$ beats/minute).The average EEEx ( $\mathrm{kcal} /$ minute) over the 15 minute interval will be calculated from measured oxygen 
consumption and carbon dioxide production using the Weir equation [23]. We have not proposed measures of EEEx during weight loss since the exercise prescription is progressive, and will be confounded by continuous changes in body weight induced by the weight loss protocol. In addition, our primary aim is to evaluate the effect of EEEx on the prevention of weight regain, not on weight loss.

\subsection{Behavioral strategies for increasing exercise compliance}

We will employ behavioral strategies to increase exercise compliance that are based on wellaccepted theoretical models including SCT, Problem Solving Theory, Relapse Prevention and Stages of Motivational Readiness for change, to provide participants the necessary skills to adopt and maintain the targeted exercise behaviors [52-56]. Specific strategies will include, but not be limited to, self-monitoring, goal setting, problem solving, mastery skills, social support, and relapse prevention. In the context of this investigation, goals for the level of exercise will be predetermined by study design, with strategies implemented by exercise facility staff to reinforce the attainment of these prescribed goals.

To facilitate adoption of prescribed doses of exercise the prescription will be progressed slowly (Table 3 ) to provide mastery experiences and improve self-efficacy as participants move from a sedentary state to 100 and then to, 150,225 or $300 \mathrm{~min} /$ week, during the first 3 to 6 months of the intervention. Participants will also be provided access to an exercise facility that is easily accessible (on-site free parking), temperature controlled, and with stateof-the-art equipment and entertainment packages. In addition, during the first 6 months of the study we will provide the opportunity for participants to complete one of their supervised exercise sessions prior to/following their regular face-to-face clinic meetings which will reduce participant burden and may improve exercise compliance.

\subsection{Strategies for participant retention}

We will obtain participant contact information to include name, address, telephone numbers, and email, as well as contact information for at least two family members or close friends who will know the location of the participant throughout the duration of the intervention. Participants who miss more than 3 consecutive scheduled on-site exercise sessions will be contacted by study staff by phone, text or email. After a maximum of 3 unsuccessful contact attempts, no further attempts will be made during the intervention period. However, participants as well as family members will be contacted to encourage completion of the end-study outcome assessments. Staff training will focus on relationship building between participants and the intervention team. Weekly project meetings with the principal investigator and the intervention team will problem solve any participant retention issues that may arise. We will also mail birthday cards, holiday cards, and program reminders to all participants. Undeliverable mailings, returned with a forwarding address, will be used to track participants who were potentially lost to follow-up. We will use behavioral contracts at multiple time points during recruitment, assessment, and after randomization to support our retention efforts and to determine the understanding of participants regarding the requirements for study participation. These strategies resulted in a loss to follow-up of $22 \%$ in our recently completed trial comparing the effectiveness of phone with face-to-face behavioral clinics for weight management [45]

\subsection{Outcome assessments}

All outcome assessments will be completed by trained research staff in the Center for Physical Activity and Weight Management, Energy Balance Laboratories (EBL) at either The University of Kansas-Lawrence or the University of Kansas Medical Center, Kansas City, Kansas. Research staff performing outcome assessments will be separate from those 
who supervise exercise or conduct behavioral clinics and will be blinded to study condition. The schedule for outcome assessments is presented in Table 3.

2.13.1. Anthropometrics-Body weight, height and waist circumference will be recorded at $-3,0,3,6,9$ and 12 months. Weight will be obtained using a digital scale accurate to $\pm 0.1 \mathrm{~kg}$ (Befour Inc. Model \#PS6600, Saukville, WI). Participants will report to the EBL between the hours of 6 and $10 \mathrm{AM}$, after an overnight fast, and be weighted prior to breakfast after attempting to void wearing a standard hospital gown. Height will be measured using a stadiometer (Model PE-WM-60-84, Perspective Enterprises, Portage, MI) and BMI $\left(\mathrm{kg} / \mathrm{m}^{2}\right)$ will be calculated. Waist circumference will be assessed using procedures described by Lohman et al. [41]. We will obtain 2 measurements per site within $2 \mathrm{~cm}$.

2.13.2. Energy intake-Energy intake will be assessed by 3 -day food records ( 2 week days/1 weekend day) at $-3,0,3,6,9$ and 12 months prior to reporting to the laboratory for anthropometric assessments. Participants will be given verbal instructions and provided with written instructions titled "How to complete your food record" to improve record keeping. Food records will be reviewed by a registered dietitian during the laboratory visit to clarify any ambiguities. Data from the 3-day food records will be entered in the Nutrition Data System for Research (NDSR, version 2012, University of Minnesota) for calculation of energy and macronutrient content.

2.13.3 Dietary staff training and quality control-All staff will complete standardized training for 24-hour recalls and NDS-R coding, prior to the beginning of data collection, with refresher sessions every 2 months, thereafter. After initial training, all dietary assessment staff will be required to complete ten 24-hour recalls obtained from nonstudy subjects and enter this data directly into NDS-R. The recalls will be evaluated according to a published dietary recall documentation checklist [51]. An error rate of less than $5 \%$ on this checklist and on NDS-R coding will be required before interviewers will be allowed to collect and process dietary recall data. During the study, all dietary recalls will be evaluated by our study dietician using the recall documentation checklist before entry into the study database. Any recall with greater than 5\% error will be eliminated and another recall obtained. Study staff demonstrating an error rate of $5 \%$ or greater for either energy intake or macronutrient composition, will be required to obtain further training and repeat assessment of accuracy described previously. Staff not meeting our criteria for accuracy at any time during the study following 3 attempts will not be permitted to collect or process dietary data.

2.13.4. Physical activity by accelerometry-To determine day-to-day and within-day variation in PA (i.e., compare exercise to non-exercise days, exercise time vs. non-exercise time), as well as to estimate the time spent in a range of PA intensity levels (moderate, vigorous), participants will wear an ActiGraph Model GT3+ (ActiGraph, LLC, Pensacola, FL) portable accelerometer for 7 consecutive days at $-3,0,6$, and 12 months on a belt over the non-dominant hip. The data collection interval will be set at one min with a minimum of 12 hours constituting a valid monitored day. We will apply the intensity cut-points used in the National Health and Nutrition Examination Survey as described by Troiano et al [57]; moderate $(>3 \mathrm{METS}=>2020$ counts $/ \mathrm{min})$, vigorous $(>6 \mathrm{METS}=>5999$ counts $/ \mathrm{min})$. We have a custom SAS program to complete these analyses.

2.13.5. Process Measures: Diet/exercise-Process data will be collected to assess the fidelity of both the dietary and exercise components of the intervention. Diet: Health educators will track the number of PM's consumed, fruit and vegetable intake, attendance at clinic meetings, and the number of reported midweek checks completed. Exercise: Staff will 
track the number of both supervised and unsupervised sessions completed and the intensity and duration of all completed sessions.

2.13.6. Medical management-Signed clearance from a licensed physician will be required prior to participation. Additionally, all individuals must qualify based on their health history reported at baseline.

\subsection{Analysis plan and statistical power}

2.14.1. Analysis plan aims 1 and 2-1) To assess the effectiveness of 3 exercise recommendations on the prevention of weight regain over 12 mos. subsequent to clinically significant weight loss ( $\geq 5 \%)$; and 2) To evaluate gender differences in weight regain in response to 3 exercise recommendations. To evaluate the significance of the main effects (treatment and/or gender) on weight change (month 12 - month 0 ) we will use a 2 -factor analysis of variance to compare weight change during the weight regain prevention intervention while including the treatment $\mathrm{X}$ gender interaction term in the initial model. If the interaction term is not significant, as expected, it will be excluded and the model will include only main effects for treatment and gender. If Treatment effect is significant $(p<0.05)$, we will conduct pairwise comparisons between the 3 treatment groups using a type I error rate of 0.0167 for each pairwise comparison. The test of the main effect of gender involves only one comparison and thus will be evaluated with a type 1 error rate of 0.05 . We will then use linear mixed models, assuming an autoregressive correlation structure over time, to model weight at 3, 6,9 and 12 months post randomization, using weight at time 0 as a covariate. We will include main effects (treatment, gender, time) and also examine potential main effect by time interactions. This analysis will allow us to determine the time course of change, evaluate when treatment differences occur, and determine if treatment differences continue to increase or attenuate over time. We will also determine at what time point gender differences occur and whether or not differences increase or attenuate over time.

We will determine if proportion of participants lost to follow-up differs by treatment and/or gender and compare demographic characteristics between those lost to follow-up and completers. If the proportion lost to follow-up differs across treatment groups, we will examine demographic characteristics (gender, age, amount of weight loss) between lost to follow-up and completers) then determine if there are between group differences in those characteristics. If missing data are related to treatment and/or demographic characteristics, missing data will be imputed with model based multiple imputation methods, if not, traditional multiple imputation will be used. Based upon our previous effectiveness weight management trial, we expect $\_5 \%$ of randomized participants will be lost to follow-up [45].

\subsubsection{Analysis plan aim 3: To assess potential compensatory changes in daily PA and El on weight regain in response to 3 exercise recommendations}

-We will compare change in daily PA and EI in a similar manner as described for aims 1 and 2. We will first conduct 2-factor (treatment/gender) analysis of variance to examine changes in PA and EI over the duration of the prevention of weight regain intervention (0 to 12 months). We will then construct linear mixed models to evaluate the impact of PA and EI on weight regain while controlling for both treatment group and gender.

2.14.3 Potential exploratory analyses-Data collected in this study will provide the opportunity for a number of interesting exploratory analyses which will include by not be limited to: Dose/response: A preliminary assessment of the dose-response association between total EEEx and weight regain will be obtained by calculating the actual EEEx during the weight regain prevention intervention and using this as a covariate in a linear 
mixed model assessing weight change while controlling for gender. Efficacy: We will conduct a "per protocol/pseudo-efficacy" analysis for weight regain including only participants who complete the study and are at least $80 \%$ compliant with exercise recommendations. This analysis will be performed as described for our primary aims 1 and 2. Amount of weight loss: The amount of weight loss may impact weight regain. We will include the amount of weight loss as a covariate in our longitudinal linear mixed models to examine its impact on weight regain while controlling for treatment, gender and time. Energy intake/diet composition: To determine if energy intake and/or diet composition (\% fat, protein, carbohydrate) impact weight regain we will include these variables in a longitudinal model controlling for treatment, gender, time, and initial weight loss, if significant. All statistical analyses will be completed with SAS version 9.2 or higher.

2.14.4. Power and sample size-Table 5 details the hypothesized amount of weight regain in each of the 3 exercise groups (100, 225, 300 minutes/week) by gender during weight regain prevention ( 0 to 12 months) based on the results from our recently completed trial comparing the effectiveness of weight loss/regain between behavioral clinics delivered face-to-face vs. phone conference call which included unsupervised PA [45]. Using these assumptions we propose to randomize 86 participants who lose $25 \%$ of initial weight (43 men and 43 women) to each of the 3 groups following our 3 month weight loss intervention. This provides a total sample of 258 participants ( 129 males; 129 females). To be conservative, we estimate a total attrition during weight loss (drop out + failure to achieve $5 \%$ weight loss) of $10 \%$, thus we will enroll a total of 287 participants at baseline (-3 months). Baseline enrollment has not been inflated for potential loss to follow-up as we will use intent-to-treat as our primary analysis using imputed data for those lost to follow-up as described previously (2.14.1). Using a 2-factor analysis of variance on weight regain with a type 1 error rate of 5\% the proposed sample size will provide $99 \%$ power for overall trend across the 3 treatment groups, 98\% power for test of gender and 5\% power for interaction, assuming a common standard deviation of $6.0 \mathrm{~kg}$ for weight regain from baseline to 12 months. Based on the hypothesized gender differences (Table 4) we do not expect a statistically or clinically significant treatment by gender interaction, thus the limited power. If, as expected, the interaction term is not significant, it will be excluded from the model which will then include main effects for treatment and gender. Adjusting the type 1 error rate for 3 pairwise comparisons (0.0167) we will have over $80 \%$ power for comparisons of weight regain between the 100 vs. 225 minutes/week and 225 vs. $300 \mathrm{~min} /$ week groups, and $99 \%$ power for a pairwise comparison of the 100 vs. 300 minute/week groups. Thus, we have adequate statistical power to evaluate each main effect and all potential pairwise comparisons across the 3 treatment groups.

\subsection{Discussion}

The prevention of weight regain following weight loss is problematic. Approximately 50\% of individuals who initially lose weight will regain more than $45-75 \%$ of the weight lost within 12 to 30 months from the end of treatment $[8,12-16]$. This trial is designed and adequately powered to evaluate the level of exercise associated with the prevention of weight regain subsequent to clinically significant weight loss. Important design features of this study include: randomization subsequent to weight loss to assure equal distribution of physiologic changes associated with weight loss that may impact weight regain across exercise groups; verification of completion of all exercise sessions and the assessment and documentation of EEEx; adequate statistical power to evaluate gender differences in the response to 3 levels of exercise; and a long duration intervention (12 months) with assessment of primary outcomes at 3 month intervals to examine the time course of weight change. The results of this trial will provide a more complete understanding of the weight change response to exercise following weight loss in both men and women and will provide 
preliminary information regarding compensatory changes in energy intake and daily PA that may affect this response. This information will be important for the development of evidenced based recommendations for the level of EEEx associated with the prevention of weight regain in men and women.

\section{Acknowledgments}

Funding: National Heart, Lung and Blood Institute (RO1-11842)

NCT registration NCT01664715

\section{Abbreviations}

$\begin{array}{ll}\text { ACSM } & \text { American College of Sports Medicine } \\ \text { EBL } & \text { Energy Balance Laboratory } \\ \text { EI } & \text { Energy intake } \\ \text { EEEx } & \text { Energy expenditure of exercise } \\ \text { HR } & \text { Heart rate } \\ \text { IOM } & \text { Institute of Medicine } \\ \text { NDS-R } & \text { Nutrition Data Systems for Research } \\ \text { PA } & \text { Physical activity } \\ \text { PCP } & \text { Primary care provider } \\ \text { PM's } & \text { Pre-packaged meals } \\ \text { RMR } & \text { Resting metabolic rate } \\ \text { SCT } & \text { Social Cognitive Theory }\end{array}$

\section{References}

[1]. Stevens VJ, Obarzanek E, Cook NR, Lee IM, Appel LJ, Smith West D, et al. Long-term weight loss and changes in blood pressure: results of the Trials of Hypertension Prevention, Phase II. Ann Intern Med. 2001; 134:1-11. [PubMed: 11187414]

[2]. Knowler WC, Barrett-Connor E, Fowler SE, Hamman RF, Lachin JM, Walker EA, et al. Reduction in the incidence of type 2 diabetes with lifestyle intervention or metformin. $\mathrm{N}$ Engl $\mathrm{J}$ Med. 2002; 346:393-403. [PubMed: 11832527]

[3]. Tuomilehto J, Lindstrom J, Eriksson JG, Valle TT, Hamalainen H, Ilanne-Parikka P, et al. Prevention of type 2 diabetes mellitus by changes in lifestyle among subjects with impaired glucose tolerance. N Engl J Med. 2001; 344:1343-50. [PubMed: 11333990]

[4]. Whelton PK, Appel LJ, Espeland MA, Applegate WB, Ettinger WH Jr. Kostis JB, et al. Sodium reduction and weight loss in the treatment of hypertension in older persons: a randomized controlled trial of nonpharmacologic interventions in the elderly (tone). JAMA. 1998; 279:839_ 46. [PubMed: 9515998]

[5]. Network, W-cI. Weight loss for life. NIH Publication No 04-3700. 2009; http://win.niddk.nih.gov/ publications/PDFs/WeightLossforLife_04.pdf

[6]. Expert panel on the identification evaluation and treatment of overweight in adults. Clinical guidelines on the identification, evaluation, and treatment of overweight and obesity in adults: executive summary. Am J Clin Nutr. 1998; 68:899-917. [PubMed: 9771869]

[7]. Goodrick GK, Foreyt JP. Why treatments for obesity don't last. J Am Diet Assoc. 1991; 91(10): 1243-7. [PubMed: 1918743] 
[8]. Foreyt JP, Goodrick GK. Evidence for success of behavior modification in weight loss and control. Ann Intern Med. 1993; 119:698-701. [PubMed: 8363200]

[9]. Racette S, Schoeller D, Kushner R, Neil K, Herling-Iaffaldano K. Effects of aerobic exercise and dietary carbohydrate on energy expenditure and body composition during weight reduction in obese women. Am J Clin Nutr. 1994; 61:486-94. [PubMed: 7872211]

[10]. Meckling KA, O'Sullivan C, Saari D. Comparison of a low-fat diet to a low-carbohydrate diet on weight loss, body composition, and risk factors for diabetes and cardiovascular disease in freeliving, overweight men and women. J Clin Endocrinol Metab. 2004; 89:2717-23. [PubMed: 15181047]

[11]. Flegal KM, Carroll MD, Kit BK, Ogden CL. Prevalence of Obesity and Trends in the Distribution of Body Mass Index Among US Adults, 1999-2010. Journal of the American Medical Association. 2012; 307:491-7. [PubMed: 22253363]

[12]. Barte JC, Ter Bogt NC, Bogers RP, Teixeira PJ, Blissmer B, Mori TA, et al. Maintenance of weight loss after lifestyle interventions for overweight and obesity, a systematic review. Obes Rev. 2010; 1:899-906. [PubMed: 20345430]

[13]. Borg P, Kukkonen-Harjula L, Fogelholm M, Pasanen M. Effects of walking or resistance training on weight loss maintenance in obese, middle-aged men: a randomized trial. Int J Obes. 2002; 26:676-83.

[14]. Toubro S, Astrup A. Randomised comparison of diets for maintaining obese subjects' weight after major weight loss: ad lib, low fat, high carbohydrate diet v fixed energy intake. BMJ. 1997; 314:29-34. [PubMed: 9001476]

[15]. Weiss EC, Galuska DA, Kettel Khan L, Gillespie C, Serdula MK. Weight regain in U.S. adults who experienced substantial weight loss, 1999-2002. Am J Prev Med. 2007; 33:34-40. [PubMed: 17572309]

[16]. Phelan S, Wing RR, Loria CM, Y K, Lewis CE. Prevalance and predictors of weight-loss maintenance in a biracial cohort: Results from the Coronary Artery Risk Development in Young Adults Study. Am J Prev Med. 2010; 39:546-54. [PubMed: 21084075]

[17]. Blundell JE, Gillett A. Control of food intake in the obese. Obes Res. 2001; 9(Suppl 4):263S70S. [PubMed: 11707552]

[18]. Cornier MA, Grunwald GK, Johnson SL, Bessesen DH. Effects of short-term overfeeding on hunger, satiety, and energy intake in thin and reduced-obese individuals. Appetite. 2004; 43:2539. [PubMed: 15527927]

[19]. Leibel RL, Rosenbaum M, Hirsch J. Changes in energy expenditure resulting from altered body weight. N Engl J Med. 1995; 332:621-8. [PubMed: 7632212]

[20]. Rosenbaum M, Goldsmith R, Bloomfield D, Magnano A, Weimer L, Heymsfield S, et al. Lowdose leptin reverses skeletal muscle, autonomic, and neuroendocrine adaptations to maintenance of reduced weight. J Clin Invest. 2005; 115:3579-86. [PubMed: 16322796]

[21]. Doucet E, Imbeault P, St-Pierre S, Almeras N, Mauriege P, Despres J, et al. Greater than predicted decrease in energy expenditure during exercise after body weight loss in obese men. Clin Sci. 2003:89-95. [PubMed: 12617720]

[22]. Redman LM, Heilbronn LK, Martin CK, de Jonge L, Williamson DA, Delany JP, et al. Metabolic and behavioral compensations in response to caloric restriction: implications for the maintenance of weight loss. PLoS One. 2009; 4:e4377. [PubMed: 19198647]

[23]. Schwartz A, Doucet E. Relative changes in resting energy expenditure during weight loss: a systematic review. Obes Rev. 2010; 11:531-47. [PubMed: 19761507]

[24]. Saris WH, Blair SN, van Baak MA, Eaton SB, Davies PS, Di Pietro L, et al. How much physical activity is enough to prevent unhealthy weight gain? Outcome of the IASO 1st Stock Conference and consensus statement. Obes Rev. 2003; 4:101-14. [PubMed: 12760445]

[25]. Institute of Medicine. Dietary reference intake for energy, carbohydrate, fiber, fat, fatty acids, cholesterol, protein and amino acids. Washington National Academic Press; 2002.

[26]. Donnelly JE, Blair SN, Jakicic JM, Manore MM, Rankin JW, Smith BK. American College of Sports Medicine Position Stand. Appropriate physical activity intervention strategies for weight loss and prevention of weight regain for adults. Med Sci Sports Exerc. 2009; 41:459-71. [PubMed: 19127177] 
[27]. Physical activity guidelines advisory committee report to the Secretary of Health and Human Services. 2008. http:www.health.gov/PAGuidelines/committeereport.aspx

[28]. Fogelholm M, Kukkonen-Harjula K. Does physical activity prevent weight gain--a systematic review. Obes Rev. 2000; 1:95-111. [PubMed: 12119991]

[29]. American College of Sports Medicine Resources Manual for Guidelines for exercise testing and prescription. 3ed.. Williams and Wilkins; Baltimore: 2000.

[30]. Field AE, Coakley EH, Must A, Spadano JL, Laird N, Dietz WH, et al. Impact of overweight on the risk of developing common chronic diseases during a 10-year period. Archives of Internal Medicine. 2001; 161:1581-6. [PubMed: 11434789]

[31]. Mekary RA, Feskanich D, Malspeis S, Hu FB, Willett WC, Field AE. Physical activity patterns and prevention of weight gain in premenopausal women. Int J Obes (Lond). 2009

[32]. Klem ML, Wing RR, McGuire MT, Seagle HM, Hill JO. A descriptive study of individuals successful at long-term maintenance of substantial weight loss. Am J Clin Nutr. 1997; 66:23946. [PubMed: 9250100]

[33]. Physical activity guidelines for Americans. Okla Nurse. 2008; 53:25.

[34]. U.S. Department of Agriculture, U.S. Department of Health and Human Services. [Accessed April 20, 2011] Dietary Guidelines for Americans. 2010. http://www.cnpp.usda.gov/ dietaryguidelines.htm.

[35]. Jakicic JM. Exercise in the treatment of obesity. Endocrinol Metab Clin North Am. 2003; 32:967-80. [PubMed: 14711070]

[36]. Jakicic JM, Marcus BH, Lang W, Janney C. Effect of exercise on 24-month weight loss maintenance in overweight women. Arch Intern Med. 2008; 168:1550-9. [PubMed: 18663167]

[37]. Jakicic JM, Marcus BH, Gallagher KI, Napolitano M, Lang W. Effect of exercise duration and intensity on weight loss in overweight, sedentary women: a randomized trial. JAMA. 2003; 290:1323-30. [PubMed: 12966123]

[38]. McTiernan A, Sorensen B, Irwin ML, Morgan A, Yasui Y, Rudolph RE, et al. Exercise effect on weight and body fat in men and women. Obesity. 2007; 15:1496-512. [PubMed: 17557987]

[39]. Taylor H, Jacobs D, Schucker B, Knudsen J, Leon A, Debacker G. A questionnaire for the assessment of leisure time physical activities. J Chronic Dis. 1978; 31:741-55. [PubMed: 748370]

[40]. Garner DM, Olmsted MP, Bohr Y, Garfinkel PE. The eating attitudes test: psychometric features and clinical correlates. Psychol Med. 1982; 12:871-8. [PubMed: 6961471]

[41]. Zemel MB, Donnelly JE, Smith BK, Sullivan DK, Richards J, Morgan-Hanusa D, et al. Effects of dairy intake on weight maintenance. Nutr Metab. 2008; 5:28.

[42]. LeCheminant JD, Gibson CA, Sullivan DK, Hall S, Washburn R, Vernon MC, et al. Comparison of a low carbohydrate and low fat diet for weight maintenance in overweight or obese adults enrolled in a clinical weight management program. Nutr J. 2007; 6:36. [PubMed: 17976244]

[43]. Donnelly JE, Smith BK, Dunn L, Mayo MS, Jacobsen DJ, Stewart EE, et al. Comparison of a phone vs clinic approach to achieve 10\% weight loss. Int J Obes. 2007; 31:1270-6.

[44]. Bandura, A. Social Foundations of Thought and Action: A Social Cognitive Theory. Englewood Cliffs; New Jersey: Prentice-Hall: 1986.

[45]. Donnelly JE, Goetz J, Gibson C, Sullivan DK, Lee R, Smith BK, et al. Equivalent weight loss for weight management programs delivered by phone and clinic. Obesity. accepted.

[46]. LeCheminant JD, Jacobsen DJ, Hall MA, Donnelly JE. A comparison of meal replacements and medication in weight maintenance after weight loss. J Am Coll Nutr. 2005; 24:347-53. [PubMed: 16192259]

[47]. Fabian C, Klemp J, Kimler B, Aversman S, Phillips T, Zalles C, et al. Effect of successful weight loss program on biomarkers for breast cancer in postmenopausal high risk women. J Clin Oncol. 2010; 28 Abstr 1522.

[48]. Mifflin MD, St Jeor ST, Hill LA, Scott BJ, Daugherty SA, Koh YO. A new predictive equation for resting energy expenditure in healthy individuals. Am J Clin Nutr. 1990; 51:241-7. [PubMed: 2305711] 
[49]. Garber CE, Blissmer B, Deschenes MR, Franklin B, Lamonte MJ, Lee IM, et al. Quantity and Quality of Exercise for Developing and Maintaining Cardiorespiratory, Musculoskeletal, and Neuromotor Fitness in Apparently Healthy Adults: Guidance for Prescribing Exercise. Medicine \& Science in Sports \& Exercise. 2011; 43:1334-59. [PubMed: 21694556]

[50]. Donnelly JE, Washburn RA, Smith BK, Sullivan DK, Gibson C, Honas JJ, et al. A randomized, controlled, supervised, exercise trial in young overweight men and women: The Midwest Exercise Trial II (MET2). Contemp Clin Trials. 2012

[51]. Donnelly JE, Hill JO, Jacobsen DJ, Potteiger J, Sullivan DK, Johnson SL, et al. Effects of a 16month randomized controlled exercise trial on body weight and composition in young, overweight men and women: the Midwest Exercise Trial. Arch Intern Med. 2003; 163:1343-50. [PubMed: 12796071]

[52]. Bandura A. Self-efficacy: toward a unifying theory of behavior change. Psych Review. 1977; 84:191-215.

[53]. Bandura A. Health promotion by social cognitive means. Health Educ Behav. 2004; 31:143-64. [PubMed: 15090118]

[54]. Marlatt, G.; Gordon, J. Relapse prevention: maintenance strategies in the treatment of addictive behavior. Guilford Press; New York: 1985.

[55]. Prochaska JO, Velicer WF, Rossi JS, Goldstein MG, Marcus BH, Rakowski W, et al. Stages of change and decisional balance for 12 problem behaviors. Health Psych. 1994; 13(1):39-46.

[56]. Prochaska JO, Marcus BH. The transtheoretical model: applications to exercise. Exercise Adherence II. 1998

[57]. Troiano RP, Berrigan D, Dodd KW, Masse LC, Tilert T, McDowell M. Physical activity in the United States measured by accelerometer. Med Sci Sports Exerc. 2008; 40:181-8. [PubMed: 18091006]

[58]. American College of Sports Medicine Guidelines for exercise testing and prescription. 6ed.. Lippincott Williams \& Wilkins; Philadelphia: 2000. 
Table 1

Clinic Topics

\begin{tabular}{|c|c|c|c|}
\hline & & Lesson Title & Focus \\
\hline & Intro & $\begin{array}{l}\text { Program Introduction: Intro to diet, meeting } \\
\text { format, group members }\end{array}$ & Diet/Exercise/Behavior \\
\hline & 1 & Diet Review and Benefits of Exercise & Diet/Exercise \\
\hline & 2 & $\begin{array}{l}\text { Goal Setting, Realistic Weight Loss Goals, } \\
\text { Personal Reasons for Weight Loss and setting } \\
\text { goals for Exercise }\end{array}$ & Behavior \\
\hline & 3 & $\begin{array}{l}\text { Increasing Fruits and Vegetables/Benefits of } \\
\text { Fiber }\end{array}$ & Diet \\
\hline & 4 & $\begin{array}{l}\text { Exercise } 101 \text { (clothing, distractions, adverse } \\
\text { weather, etc.) }\end{array}$ & Exercise \\
\hline Weight Loss (FTF) & 5 & Self-Monitoring of diet and exercise behaviors & Diet/Exercise \\
\hline Phase & 6 & $\begin{array}{l}\text { Know Your Health Numbers (effects of nutrient } \\
\text { dense diet and PA on metabolic risk factors) }\end{array}$ & Diet/Exercise \\
\hline & 7 & $\begin{array}{l}\text { Reading Food Labels - Your Tool to Healthy } \\
\text { Eating }\end{array}$ & Diet \\
\hline & 8 & Warm-up/Cool-down and Injury prevention & Exercise \\
\hline & 9 & $\begin{array}{l}\text { Making Exercise Part of Your Routine (i.e., } \\
\text { planning, adherence, and coping with sore } \\
\text { muscles) }\end{array}$ & Exercise \\
\hline & 10 & F.I.T (Frequency, Intensity, and Duration) & Exercise \\
\hline & 11 & Healthy cooking and Meal Planning & Diet \\
\hline & 12 & $\begin{array}{l}\text { Personal Plan (maintenance calories and } \\
\text { approved entrees) }\end{array}$ & Diet \\
\hline & 13 & $\begin{array}{l}\text { Review of Program Goals/Objectives } \\
\text { Exercise Adherence (importance of attendance } \\
\text { and coping with life events) }\end{array}$ & Behavior/Exercise \\
\hline & 14 & Intuitive eating & Diet/Behavior \\
\hline & 15 & Grocery Shopping & Diet \\
\hline & 16 & Portion distortion & Diet \\
\hline Weight reagin nrevention & 17 & Maintaining motivation for diet and exercise & Diet/exercise \\
\hline Month 0-3 & 18 & Exercising on vacation & Exercise \\
\hline & 19 & Environmental Control & Behavior \\
\hline & 20 & Eating on the Go / Hidden calories & Diet \\
\hline & 21 & Resistance Training & Exercise \\
\hline & 22 & Emotional Eating & Diet/Behavior \\
\hline & 23 & Energy Density & Diet \\
\hline & 24 & Circuit Training (With demo) & Exercise \\
\hline & 26 & Doing exercise on your own & Exercise \\
\hline & 28 & Social Relationships and Social Situations & Behavior \\
\hline $\begin{array}{l}\text { Weight regain prevention Month 3-12 } \\
\text { (Phone) }\end{array}$ & 30 & $\begin{array}{l}\text { Benefits of Exercise (i.e., general physiology and } \\
\text { diseases) }\end{array}$ & Exercise \\
\hline & 32 & $\begin{array}{l}\text { Mental Traps, Cognitive Attributions \& } \\
\text { Reframing }\end{array}$ & Behavior \\
\hline
\end{tabular}




\begin{tabular}{|c|l|l|}
$\mathbf{3 4}$ & Myth Busters- Exercise & Exercise \\
\hline $\mathbf{3 6}$ & $\begin{array}{l}\text { Coping with Cravings \&Stress Related Eating } \\
\text { (Urge surfing) }\end{array}$ & Diet/Behavior \\
\hline $\mathbf{3 8}$ & $\begin{array}{l}\text { Increasing Lifestyle Activities and Decreasing } \\
\text { Sedentary Time }\end{array}$ & Exercise \\
\hline $\mathbf{4 0}$ & Mindful Eating & Diet \\
\hline $\mathbf{4 2}$ & Myth Busters - Diet and fad diets & Diet \\
\hline $\mathbf{4 4}$ & Exercise Variety & Exercise \\
\hline $\mathbf{4 6}$ & $\begin{array}{l}\text { Evidence Based Advice on Eating for Long-term } \\
\text { Weight Control }\end{array}$ & Diet/Behavior \\
\hline $\mathbf{4 8}$ & Healthy Breakfast Tips/ Picking Healthy Snacks & Diet \\
\hline $\mathbf{5 0}$ & Energy expenditure and metabolism & Exercise \\
\hline $\mathbf{5 2}$ & $\begin{array}{l}\text { Psychosocial Barriers to Weight Maintenance \& } \\
\text { Self and Body Image }\end{array}$ & Behavior \\
\hline $\mathbf{5 4}$ & $\begin{array}{l}\text { Accountability and Self-Monitoring Revisited - } \\
\text { Looking towards the future }\end{array}$ & Behavior \\
\hline $\mathbf{5 6}$ & Meal Planning Revisited & Diet \\
\hline $\mathbf{5 8}$ & $\begin{array}{l}\text { Successful Weight Management Tips \& Strategies/Relapse } \\
\text { Prevention Revisited }\end{array}$ & Diet/Exercise/Behavior \\
\hline $\mathbf{6 0}$ & Reflecting and Re-Motivating and Reassessing Goals & Behavior \\
\hline
\end{tabular}




\section{Table 2}

Exercise progression (minutes/week) during the prevention of weight regain (weeks 13-60) for the 3 exercise groups

\begin{tabular}{|l|l|l|l|}
\hline & \multicolumn{3}{|c|}{ Treatment Groups } \\
\hline Study Week & $\mathbf{1 5 0} \mathbf{~} \mathbf{m}$ /wk. & $\mathbf{2 2 5} \mathbf{~ \mathbf { n }} / \mathbf{w k}$. & $\mathbf{3 0 0} \mathbf{~ \mathbf { n }} / \mathbf{w k}$. \\
\hline 13 & 125 & 125 & 175 \\
\hline 14 & 130 & 135 & 200 \\
\hline 15 & 135 & 150 & 225 \\
\hline 16 & 140 & 175 & 250 \\
\hline 17 & 145 & 200 & 275 \\
\hline $18-60$ & 150 & 225 & 300 \\
\hline
\end{tabular}


Table 3

Outcome assessment schedule

\begin{tabular}{|c|c|c|c|c|c|c|}
\hline \multirow[b]{2}{*}{ Variable } & \multicolumn{6}{|l|}{ Time Points } \\
\hline & -3 months & 0 months & 3 months & 6 months & 9 months & 12 months \\
\hline $\begin{array}{l}\text { Anthropometrics } \\
\text { Weight } \\
\text { Height } \\
\text { Waist Circumference }\end{array}$ & $\begin{array}{l}X \\
X \\
X\end{array}$ & $\begin{array}{l}X \\
X \\
X\end{array}$ & $\begin{array}{l}X \\
X \\
X\end{array}$ & $\begin{array}{l}X \\
X \\
X\end{array}$ & $\begin{array}{l}X \\
X \\
X\end{array}$ & $\begin{array}{l}X \\
X \\
X\end{array}$ \\
\hline DXA & $\mathrm{X}$ & $\mathrm{X}$ & & $\mathrm{X}$ & & $\mathrm{X}$ \\
\hline $\begin{array}{l}\text { Energy Intake } \\
\text { (3-day food records) }\end{array}$ & $\mathrm{X}$ & $\mathrm{X}$ & $\mathrm{X}$ & $\mathrm{X}$ & $\mathrm{X}$ & $\mathrm{X}$ \\
\hline Fitness & $\mathrm{X}$ & $\mathrm{X}$ & & $\mathrm{X}$ & & $\mathrm{X}$ \\
\hline $\begin{array}{l}\text { Physical Activity } \\
\text { (Accelerometer) }\end{array}$ & $\mathrm{X}$ & $\mathrm{X}$ & & $\mathrm{X}$ & & $\mathrm{X}$ \\
\hline
\end{tabular}


Table 4

Weight change $(\mathrm{kg})$ over the weight regain prevention intervention

\begin{tabular}{|l|c|c|c|}
\hline & \multicolumn{3}{|c|}{ Treatment Groups } \\
\hline & $\mathbf{1 5 0}$ & $\mathbf{2 2 5}$ & $\mathbf{3 0 0}$ \\
\hline $\begin{array}{l}\text { Overall change } \\
(\mathrm{kg})\end{array}$ & 8.0 & 5.0 & 2.0 \\
\hline $\begin{array}{l}\text { Female change } \\
(\mathrm{kg})\end{array}$ & 9.6 & 6.5 & 3.5 \\
\hline Male change $(\mathrm{kg})$ & 6.4 & 3.5 & 0.5 \\
\hline
\end{tabular}

